\title{
Unusual Metastases from a Pleomorphic Adenoma of the Parotid Gland: A Case and Literature's Review
}

\author{
Giuditta Mannelli", Lucio Rucci, Oreste Gallo \\ First Clinic of Otorhinolaryngology-Head and Neck Surgery, University of Florence, Florence, Italy \\ Email: *mannelli.giuditta@gmail.com
}

Received October 19, 2012; revised November 20, 2012; accepted December 5, 2012

Copyright (c) 2013 Giuditta Mannelli et al. This is an open access article distributed under the Creative Commons Attribution License, which permits unrestricted use, distribution, and reproduction in any medium, provided the original work is properly cited.

\begin{abstract}
Background: Pleomorphic adenoma constitutes the most common benign parotid gland tumor. Local recurrence after surgery has been described in $1 \%-5 \%$ of cases, and malignant evolution in $2 \%-9 \%$ of cases. Metastasizing pleomorphic adenomas has rarely been reported. No histologic or molecular parameters exist at the present time that could predict the development of metastasis in these neoplasms. Methods and Results: A 54-year-old woman had a superficial left parotidectomy with facial nerve preservation for pleomorphic adenoma. She developed two recurrences in 2000 and 2001, but 25 years later the first operation she presented a metastasis in her right gluteal region with evidence of local recurrence. Conclusion: Metastatizing pleomorphic adenoma is a rare pathologic situation but, in case of swelling or lump appearance in patients with pleomorphic adenoma history, it should be taken in mind.
\end{abstract}

Keywords: Salivary Gland Tumors; Pleomorphic Adenoma; Metastases; Surgery; Gluteal Region

\section{Introduction}

Pleomorphic adenoma is the most common parotid tumor in both adult and pediatric populations. It is also termed mixed tumor and it is generally accepted as biologically benign, showing a slow-growing and well-circumscribed margin. By definition, this tumor does not metastasize at all. However, tumor recurrence and/or malignant degeneration are known as possible complications. A rare and controversial clinical entity is a metastasizing pleomorphic adenoma which, although histologically identical to pleomorphic adenoma, metastasizes to regional and distant sites, usually after many years, and after treatment, without demonstrating any malignant histopathological features. Authors such as Wenig [1] suggest that Metastatic Mixed Tumors appear after multiple recurrences of an original pleomorphic adenoma in the primary site, mainly because of many inappropriate surgical manipulations that could promote the development of disseminating metastasis. Although apparently defined by pathologists as benign, mortality can be as high as $22 \%$, according to Ellis and Auclair [2]. Metastasizing pleomorphic adenoma has neither age or gender predilection, nor association with race or geographic location [3]. This tumor usually presents multiple local recurrences, at least

\footnotetext{
"Corresponding author.
}

two or more before the development of metastatic foci [1]. Nevertheless, many reported cases in literature are without prior evidence of local recurrence [4,5]. The interval between diagnoses of the primary tumor and metastases ranges from 3 to 52 years after the occurrence of the primary lesion. Bone is the most common site for metastases, followed by head and neck deposits such as regional lymph nodes, the oral cavity and pharynx, then skin, liver, retroperitoneum, kidney, calvarium and nervous system which are the other less interested sites of interess [3].

Here, we present a case of a parotid pleomorphic adenoma that metastasized to the right gluteal region with evidence of local recurrence.

\section{Case Report}

A 54-year-old woman presented in 1983 with a slow growing mass in tail of her left parotid gland. She underwent a superficial left parotidectomy with facial nerve preservation and the mass was histologically confirmed as pleomorphic adenoma. Unfortunately, she presented again, both in 2000 and 2001, a lesion in the surgical field, localized immediately under previous skin incision, which was excised and confirmed as a recurrent pleomorphic adenoma. During the last operation facial nerve 
was sacrificed. In January 2008, because of a recent appearance of a little, painless and smooth ovoid mass in the right gluteal region, the patient underwent ultrasonography that confirmed the presence in this region of a hypoechoic little mass, $17.4 \times 12.3 \times 16.1 \mathrm{~mm}$, with posterior wall reinforcement, showing slim, regular and not vascularized walls. These features suggested a sebaceous cyst or any dermatofibroma (Figure 1). In order to clarify the nature of the lesion, a MR (magnetic resonance) exam of the pelvis was performed in December 2008 and confirmed the presence of an extra-muscolar and subfascial mass in the right gluteal region (Figure 2). In January 2009, the patient underwent the surgical excision of the lesion and the histological investigation documented possible soft tissue's metastasis from a pleomorphic adenoma, without atypia, mytosis neither necrosis (Figure 3).

Due to her anamnestic data, the patient was revalued to investigate the presence of other distant metastasis and possible local recurrence. Therefore, in June 2009 the CT scans excluded metastasis at the chest, while the CT scans of head and neck evidenced the presence of a mass under the left petrosa which was penetrating the mastoid cells and was in contact with the labyrinthine system (Figures 4(a) and (b)).

This was compatible with a recurrence of pleomorphic adenoma. Even though imaging techniques spoke about a local recurrence, patient did not complain any symptoms at all before. Except for dizziness, confirmed by a vestibular exam suggesting for the presence of a central vestibular disorder. The patient had normal hearing.

\section{Discussion}

Since the 1940s a rare and unusual entity, distinct from pleomorphic adenoma, has been recognised as its malignant variants: metastasizing pleomorphic adenoma (MPA). This one appears as a histologically benign lesion metastasizes to areas including skin (scapular region etc.), cervical lymph nodes, paranasal sinuses, lungs, liver, kidney, vertebrae and long bones, external auditory canal and larynx. Classification of salivary gland tumors by Foote and Frazell in 1953 [6] gives insufficient clinical or histological information about MPA; by reviewing cases reported in literature is possible to make important inferences about behavior and outcome of patients presenting with enigmatic conditions. In fact, between 1953 and 2012, 57 global cases of MPA have been reported in literature. The head and neck appears like the most common site for metastasis (43.8\%), followed by lungs (33.3\%), bones (33.3\%), and abdominal viscera (10.5\%). Within the head and neck, there are 11 metastases (19.3\%) to regional lymph nodes, and 14 tumor deposits (24.5\%) are detected in nonlymphatic extracranial tissues such as skin or paranasal sinuses. There is one case of

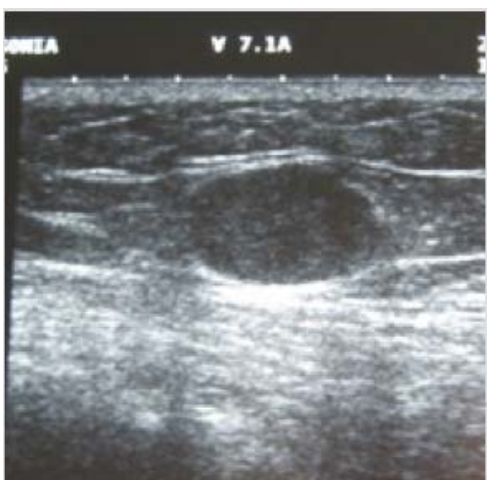

Figure 1. Ultrasonography exam of the right gluteal region which shows the presence of a hypoechoic little mass, $17.4 \times$ $12.3 \times 16.1 \mathrm{~mm}$, with posterior wall renfoircement.

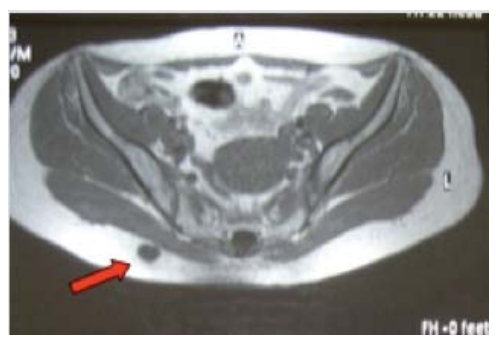

Figure 2. MR (magnetic resonance) section of the pelvis confirming the presence of an extra-muscolar and subfascial mass in the right gluteal region.

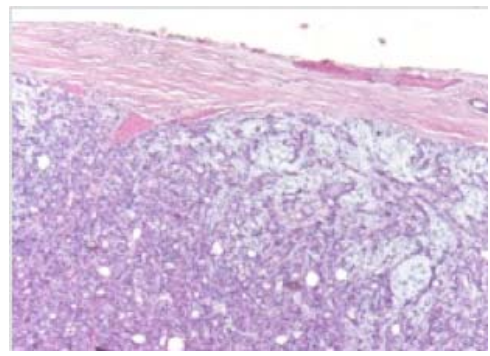

Figure 3. Histological exams documents pleomorphic adenoma's soft tissue metastasis nature.

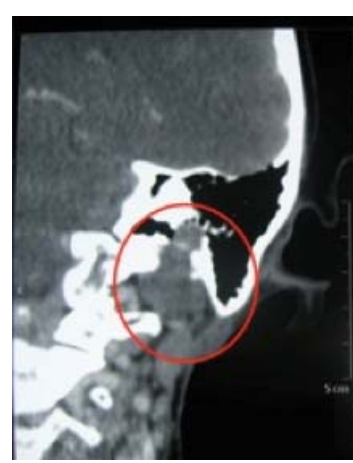

(a)

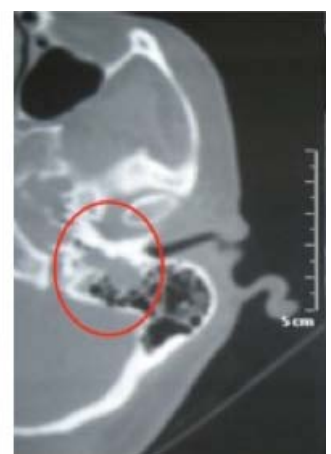

(b)
Figure 4. CT (Computerized tomography) scans of head and neck evidencing: (a) the presence of a mass under the left petrosa penetrating the mastoid cells and (b) being in contact with the labyrinthine system. 
intracranial metastases. All these cases derived from the summation of the cases reviewed by Nouraei et al. [7] and the new ones found in literature from 2005 to the entire 2009. By comparing the most common location for metastasis, the head and neck site has become the first one interested by metastases of MPA, while bones from being the first location has become the third one after lungs. On Table 1, are reported several data regarding overall series.

It is rare for a tumor appearing histologically benign to have the capacity to metastasize; the metastasizing "benign" pleomorphic adenoma is considered distinct from the true malignant mixed tumor (carcinoma ex-pleomorphic adenoma and carcinosarcoma) because it remains histologically benign [7]. That is why, in case of previous benign head and neck neoplasia, the appearance of new masses is not easily linked to the previous disease, even if it should always lead towards a possible connection with the previous head and neck disease before thinking about a new disorder. The MPA is an emblematic possibility; in fact, because of its diagnosis, which is determined by histopathological analysis only, clinical examinations could steer wrongly towards differential diagnosis with many other kind of possible neoplasia depending on the site of presentation. We saw above, how head and neck is the most common site of metastases from MPA, in this case ultrasonography of the neck or computer tomography scan or magnetic resonance analysis may help in distinguishing the morphological characteristics of the new lump, especially in identifying its malignant or benign nature. The same critical attitude should be applied in case of mass in other sites. Moreover, the historical records of the patients may help in thinking about rare condition, such as the MPA. In fact, even if the mechanism for the metastatic behaviour is not clear, it seems to be more common when the original pleomorphic adenoma is uncompletely excised, due to repeated surgical manipulation in the attempt to excise recurrences that could push neoplastic cells into vascular spaces.

Here we reported the case a 54 years old woman who underwent in 1983 a superficial left parotidectomy with facial nerve preservation for a pleomorphic adenoma which recurred locally on twice without any distant metastasis and ones in 2009, 25 years later the first surgical operation at her left parotid gland, with a soft tissue metastasis. Recurrence of the tumor at the primary site occurs in about $90 \%$ of cases of metastasizing pleomorphic adenoma with a usual long interval between the diagnosis of the primary lesion and the metastasis [1]. Nouraei et al. [7] demonstrated about an $81 \%$ of local recurrence prior the distant metastasis's detection, with the mean time of presentation to recurrence of $5.0 \pm 4.9$ years. The mean \pm SD time from initial presentation to the detection
Table 1. Sites of metastasis: update of metastases' locations.

\begin{tabular}{ccc}
\hline Sites & Numbers & \% \\
Total & 57 & $100 \%$ \\
Head and Neck & & \\
Skin/scalp/skull & 11 & 19.3 \\
Cervical limph node & 11 & 19.3 \\
Paranasal sinuses & 2 & 3.5 \\
Intracranial & 1 & 1.7 \\
Visceral & & \\
Pulmonary & 19 & 33.3 \\
Renal & 4 & 7 \\
Hepatic & 2 & 3.5 \\
Mediastinal limph node & 1 & 1.7 \\
Larynx & 1 & 1.7 \\
Bone & & \\
Pelvic/sacrum & & 10.5 \\
Vertebra & 6 & 8.8 \\
Femur & 5 & 5.3 \\
Humerus & 3 & 3.5 \\
Ribs & 2 & 3.5 \\
Scapula & 2 & 1.7 \\
Skin nodules (not within head and neck) & 3 & 5.3 \\
\hline & &
\end{tabular}

of metastases who had no intervening local recurrences was $12.3 \pm 8.6$ years, while for patients with a history of local recurrence this was $16.9 \pm 13.3$ years. That is why it may also occur many years after its seemingly complete excision at the primary site. There are a few reports of cases of pleomorphic adenoma metastases without local recurrence at the primary site frequently many years after primary excision [5]. In our report the third local recurrence has been identified after the metastasis's detection, because patient did not claim any clinical symptoms at all except after CT scans immediately.

Since no clinical criteria differentiate the typical benign pleomorphic adenoma from the metastasizing one, some authors tried to identify distinguishing histologic characteristics. Wenig et al. [1] made a review of 11 cases; primary, recurrence and metastases had a similar appearance, as well as histopathological features. The primary site was recorded: eight from the parotid, two submandibular glands, and one the nasal septum. The majority had two or more recurrences before the development of their metastatic focus. The metastases were discovered at any time from 6 to 52 years after the presentation of the primary tumor. The metastatic deposits were identified in bone, lung, regional lymph nodes, skin, kidney, retroperitoneum, oral cavity, pharynx, calvarium, and brain. Mitotic rate, cellular pleomorphism, tumor infiltration, evidence of vascular or lymphatic invasion 
were compared. No differences existed. No evidence of malignancy was identified. Two of these patients died as a direct result of their metastases at 3 and 2 years, seven patients were alive without disease, and two patients died of unrelated causes. More probably, the metastatic capability reflects the accumulation of key genetic alterations, a sequential process that causes histological and biological progression [2]. El-Naggar et al. [8] considered that the lack of recognized histologic parameters of malignancy raises the possibility that a small subset of pleomorphic adenomas may have an area of submicroscopic alterations that may subsequently pursue a malignant course.

Some authors investigated the possible correlation between expression of oncogenes and tumor suppressos genes and metastasizing pleomorphic adenoma, in order to find a genetic reason of its behavior [9]. Unfortunately, no correlation has been found between specific chromosomal defects and tumor recurrence and/or metastases.

\section{Conclusions}

MPA is a low-grade malignant tumor [3], which shows the $58 \%$ disease-specific 5-years survival and about 50\% of disease-free survival rate, with a mortality rate of about 20\% [1,10]. It should not be underestimated. Multiple metastases are fatal, and their developing and presence in multiple sites, within the first 10 years after the initial diagnosis, are independent predictors of worse survival [10].

Usually, local recurrence is detected first and metastasis second, but in case of accidental metastasis in a patient with a history of pleomorphic adenoma or of its local recurrences it would be better to investigate the presence of a local recurrence, which is found in $80 \%$ of cases. We recommend, in order to prevent possible local recurrences and distant metastasis, performing superficial parotidectomy with facial nerve preservation to obtain a meticulous resection of the primary pleomorphic adenoma, which is usually curative. On the other hand, chemotherapy and radiotherapy still present a limited value [11] as well as hormonal treatment [12].

Due to the limited number of reported cases, it is difficult to understand the biology of MPA as distinct from non-MPA and to identify a specific follow-up protocol, but we agree that the metastatic disease should be treated surgically.

\section{Acknowledgements}

This manuscript is approved by all authors and all of them have participated in writing and correcting of this work. Further, all authors assure that manuscript has not been published nor is under approving by other journals or editors and they have not any conflict of interest, financial or otherwise.

\section{REFERENCES}

[1] B. M. Wenig, C. L. Hitchcok, G. L. Ellis and D. R. Gnepp, "Metastasizing Mixed Tumor of Salivary Gland: A Clinicopathologic and Flow Cytometric Analysis," American Journal of Surgical Pathology, Vol. 16, No. 9, 1992, pp. 845-858. http://dx.doi.org/10.1097/00000478-199209000-00003

[2] G. L. Ellis and P. L. Auclair, "Malignant Epithelial Tumors," In: G. L. Ellis and P. L. Auclair, Eds., Tumors of the Salivary Glands, Armed Forces Institute of Pathology, Washington DC, 1996, pp. 39-136.

[3] V. Manucha and O. B. Ioffe, "Metastasizing Pleomorphic Adenoma of Salivary Gland," Archives of Pathology \& Laboratory Medicine, Vol. 132, No. 9, 2008, pp. 14451447.

[4] G. R. Youngs and P. J. Scheuer, "Histologically Benign Mixed Parotid Tumor with Hepatic Metastasis,” Journal of Pathology, Vol. 109, No. 2, 1973, pp. 171-173. http://dx.doi.org/10.1002/path.1711090211

[5] V. Raja, C. China, K. H. Masaki and G. Nakano, "Benign Metastasizing Pleomorphic Adenoma,” Journal of Clinical Oncology, Vol. 20, No. 9, 2002, pp. 2400-2403.

[6] F. W. Foote Jr. and L. E. Frazell, "Tumors of the Major Salivary Glands,” Cancer, Vol. 6, 1953, pp. 1065-1133. http://dx.doi.org/10.1002/1097-0142(195311)6:6<1065:: AID-CNCR2820060602>3.0.CO;2-0

[7] S. A. R. Nouraei, M. S. Ferguson, P. M. Clarke, A. Sandison, G. S. Sandhu, L. Michaels and P. Rhys-Evans, "Metastasizing Pleomorphic Salivary Adenoma," Archives of Otolaryngology-Head and Neck Surgery, Vol. 132, No. 7, 2006, pp. 788-793.

http://dx.doi.org/10.1001/archotol.132.7.788

[8] A. El-Naggar, J. G. Batsakis and S. Kessler, "Benign Metastatic Mixed Tumours or Unrecognised Salivary Gland Carcinomas?” Journal of Laryngology \& Otology, Vol. 102, No. 9, 1988, pp. 810-812. http://dx.doi.org/10.1017/S0022215100106528

[9] Y. Jin, C. Jin, K. Arheden, et al., "Unbalanced Chromosomal Rearrangements in a Metastasizing Salivary Gland Tumour with Benign Histology,” Cancer Genetics and Cytogenetics, Vol. 102, No. 1, 1998, pp. 59-64. http://dx.doi.org/10.1016/S0165-4608(97)00301-4

[10] J. Klijanienko, A. El-Naggar, V. Servois, J. Rodriguez, P. Validire and P. Vielh, "Clinically Aggressive Metastasizing Pleomorphic Adenoma: Report of Two Cases,” Head and Neck, Vol. 19, No. 7, 1997, pp. 629-633.

http://dx.doi.org/10.1002/(SICI)1097-0347(199710)19:7< 629::AID-HED11>3.0.CO;2-Y

[11] J. Paris, F. Facon, M. A. Chrestian, A. Giovanni and M. Zanaret, "Recurrence of Pleomorphic Adenomas of the Parotid: Changing Attitudes," Revue de LaryngologieOtologie-Rhinologie, Vol. 124, No. 4, 2003, pp. 229-234.

[12] A. S. Glas, H. Hollema, R. E. Nap and J. T. Plukker, "Expression of Estrogen Receptor, Progesterone Receptor, and Insulin-Like Growth Factor Receptor-1 and of MIB-1 in Patients with Recurrent Pleomorphic Adenoma of the Parotid Gland,” Cancer, Vol. 94, No. 8, 2002, pp. 22112216. http://dx.doi.org/10.1002/cncr.10445 